\title{
Control of oscillators coherence by multiple delayed feedback
}

\author{
Andreas H. Pawlik ${ }^{\mathrm{a}, *}$, Arkady Pikovsky ${ }^{\mathrm{b}}$ \\ ${ }^{a}$ Leiden Observatory, PO Box 9513, 2300 RA Leiden, The Netherlands \\ ${ }^{\mathrm{b}}$ Department of Physics, University of Potsdam, 14415 Potsdam, Germany \\ Received 30 December 2005; accepted 8 May 2006 \\ Available online 15 May 2006 \\ Communicated by A.P. Fordy
}

\begin{abstract}
We demonstrate that a multiple delayed feedback is a powerful tool to control coherence properties of autonomous self-sustained oscillators. We derive the equation for the phase dynamics in presence of noise and delay, and analyze it analytically. In Gaussian approximation a closed set of equations for the frequency and the diffusion constant is obtained. Solutions of these equations are in good agreement with direct numerical simulations.
\end{abstract}

(C) 2006 Elsevier B.V. All rights reserved.

PACS: 05.40.-a; 02.50.Ey

Keywords: Phase diffusion; Delayed feedback; Control

\section{Introduction}

A delayed feedback is a general tool to control dynamics. It is used to suppress chaos (the famous Pyragas method [1], see also a review [2]) and synchronization [3,4]. For selfsustained oscillators the crucial characteristics of the dynamics is coherence, or constancy of oscillation frequency, determining their quality as clocks. As has been demonstrated in [5], the coherence of noisy self-oscillating systems changes under the influence of a single delayed feedback; for an attempt of experimental realization of such a coherence control see [6]. Based on a phase description, Goldobin et al. [5] reported both numerically and analytically on the possibility to control the coherence of the noisy Van der Pol oscillator by means of varying the feedback strength and the delay time. The main difficulty encountered in Ref. [5] was that the delay renders the oscillator dynamics non-Markovian, which makes it practically impossible to apply such well-established tools like the Fokker-Planckformalism as in the Markovian case. Therefore, they had to develop ad hoc statistical methods (Gaussian approximation).

\footnotetext{
* Corresponding author.

E-mail addresses: pawlik@strw.leidenuniv.nl (A.H. Pawlik), pikovsky@stat.physik.uni-potsdam.de (A. Pikovsky).
}

In this Letter we investigate the Van der Pol oscillator in the presence of noise and a multiple delayed feedback, thereby introducing a new control parameter in addition to the feedback strength and delay time, which enlarges the possibilities of the coherence control. Moreover, as has been demonstrated for the chaos suppression [7,8], multiple delayed feedback can significantly improve the quality of the control. As we will demonstrate below, this applies to the coherence control as well.

The Letter is structured as follows. In Section 2 we introduce our basic model. Then, in Section 3 we discuss a possibility of frequency multistability due to feedback. In Section 4 we employ the Gaussian approximation to study the diffusive properties of the model system analytically. We will find that control using a multiple delayed feedback can be superior to one with a single delayed feedback. In Section 5 we validate our results through direct numerical simulations of the model equation. Finally, we conclude with an outlook.

\section{Basic model}

The basic model we study is given by the noisy (term $\sim \zeta$ ) Van der Pol oscillator with a multiple delayed feedback with strength $k$, 


$$
\begin{aligned}
& \ddot{x}-\mu\left(1-x^{2}\right) \dot{x}+\omega_{0}^{2} x \\
& \quad=k \sum_{\nu=0}^{\infty} R^{\nu}[\dot{x}(t-(\nu+1) \tau)-\dot{x}(t-v \tau)]+\zeta(t), \\
& \left\langle\zeta(t) \zeta\left(t^{\prime}\right)\right\rangle=2 d_{\mathrm{vdP}}^{2} \delta\left(t-t^{\prime}\right) .
\end{aligned}
$$

A similar feedback has been suggested by Socolar et al. [7] in the context of chaos control. Despite the infinite sum of delays, it can be quite easily realized in experiments [8] (roughly speaking, one has to send the signal $\dot{x}(t)$ to a Fabry-Perot resonator with reflection coefficient at the 'mirrors' $R$ ). For convergence reasons we confine $R$ to the range $(-1,1)$. Noteworthy, that setting $R=0$ we recover the model with just one feedback term studied in [5].

For further considerations it is convenient to employ the so-called phase description, which is valid for small noise and feedback. For small nonlinearity $\mu$ and vanishing external force the Van der Pol oscillator has the limit cycle solution $x_{0} \approx 2 \cos \Phi, \dot{x}_{0} \approx-2 \omega_{0} \sin \Phi$, with a uniformly growing phase $\Phi(t) \approx \omega_{0} t+\Phi_{0}$. Under the influence of noise, but still in the absence of delayed feedback, the phase diffuses according to $\left\langle(\Phi(t)-\langle\Phi(t)\rangle)^{2}\right\rangle=D_{0} t$. Hereby, the uncontrolled diffusion constant $D_{0}$ is proportional to the intensity of the noise $d_{\mathrm{vdP}}^{2}$.

In the presence in Eq. (1) of small noise and weak delayed feedback, one can apply a standard procedure to derive the equation for the phase (see, e.g., $[5,9,10])$ :

$\dot{\Phi}=\omega_{0}+\frac{k}{2} \sum_{\nu=0}^{\infty} R^{\nu}\left\{\sin \left[\Phi_{(\nu+1) \tau}-\Phi\right]-\sin \left[\Phi_{\nu \tau}-\Phi\right]\right\}+\xi(t)$,

with the effective noise $\xi(t)$ satisfying $\left\langle\xi(t) \xi\left(t^{\prime}\right)\right\rangle=d^{2} \delta\left(t-t^{\prime}\right)$ and $d^{2} \equiv d_{\mathrm{vdP}}^{2} /\left(4 \omega_{0}^{2}\right)$. Here and in the following for timedelayed variables we use notations like $\Phi_{\nu \tau} \equiv \Phi(t-\nu \tau)$.

Our main goal is to investigate the diffusion properties of the phase. To this end we separate the phase into an average growth and fluctuations, $\Phi(t)=\Omega t+\Psi(t)$. We then obtain for the fluctuating instantaneous frequency $\dot{\Psi}$ by substitution into Eq. (2) the following equation

$$
\begin{aligned}
\dot{\Psi}= & \omega_{0}-\Omega+\xi \\
& +\frac{k}{2} \sum_{\nu=0}^{\infty} R^{\nu}\left\{\cos [\Omega(\nu+1) \tau] \sin \left[\Psi_{(\nu+1) \tau}-\Psi\right]\right. \\
& -\cos [\Omega \nu \tau] \sin \left[\Psi_{\nu \tau}-\Psi\right] \\
& -\sin [\Omega(\nu+1) \tau] \cos \left[\Psi_{(\nu+1) \tau}-\Psi\right] \\
& \left.+\sin [\Omega \nu \tau] \cos \left[\Psi_{\nu \tau}-\Psi\right]\right\} .
\end{aligned}
$$

\section{Noise-free case and delay-induced multistability}

Without noise, $\xi=\Psi=\dot{\Psi}=0$, Eq. (3) simplifies to $0=\omega_{0}-\Omega-\frac{k}{2} \sum_{\nu=0}^{\infty} R^{\nu}\{\sin [\Omega(\nu+1) \tau]-\sin [\Omega \nu \tau]\}$.

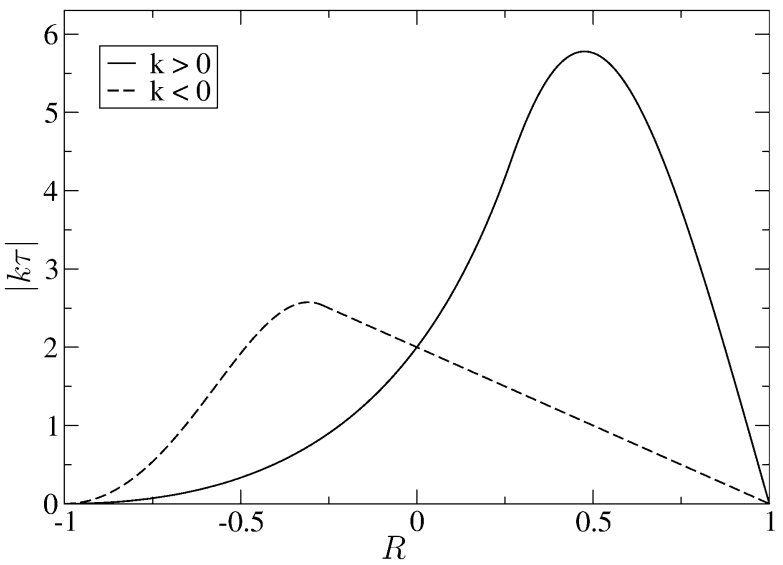

Fig. 1. Domains of absence of multistability on the plane of parameters $R-|k \tau|$.

The main effect is that the delayed feedback changes the frequency of the oscillator. Furthermore, the implicit Eq. (4) provides either a unique or multiple solutions for $\Omega$. The latter case, which we call delay-induced multistability, is especially difficult to analyze, it will be considered elsewhere. Here we choose the model parameters in the domain where no multistability occurs. The condition for Eq. (4) to have a unique solution is given by the implicit function theorem,

$\frac{\partial}{\partial \Omega}\left[\omega_{0}-\Omega-\frac{k}{2} \sum_{\nu=0}^{\infty} R^{\nu}\{\sin [\Omega(\nu+1) \tau]-\sin [\Omega \nu \tau]\}\right] \neq 0$.

Evaluating condition (5) yields the following relations the parameters $k, \tau$ and $R$ must obey to avoid multistability independent of $\omega_{0}$ :

$$
\begin{aligned}
& k \tau<\frac{2(1+R)^{2}}{1-R} \text { for } k>0 \text { and }-1<R<2-\sqrt{3}, \\
& k \tau<\frac{16(1-R) R(1+R)^{2}}{\left(1+R^{2}\right)^{2}} \text { for } k>0 \text { and } 2-\sqrt{3} \leqslant R<1, \\
& |k \tau|<\frac{16(R-1) R(1+R)^{2}}{\left(1+R^{2}\right)^{2}} \\
& \quad \text { for } k<0 \text { and }-1<R<-2+\sqrt{3}, \\
& |k \tau|<2(1-R) \quad \text { for } k<0 \text { and }-2+\sqrt{3} \leqslant R<1 .
\end{aligned}
$$

We visualize the above relations in Fig. 1. Observe that a nonzero value of $R$ can relax the restriction on the product $|k \tau|$ in comparison to the case of a single delayed feedback $(R=0)$.

\section{Statistical analysis}

Our main assumption allowing to perform statistical analysis of the basic model analytically is that the phase fluctuations $\Psi$ are Gaussian. However, we do not a priori confine the Gaussian noise $\xi$ to be white. After averaging Eq. (3) over the fluctuations of $\dot{\Psi}$ (which are Gaussian distributed, too), we obtain for the 
mean frequency $\Omega$ :

$$
\begin{aligned}
\Omega= & \omega_{0}+\frac{k}{2} \sum_{\nu=0}^{\infty} R^{\nu}\left\{\sin [\Omega \nu \tau] \mathrm{e}^{-\left\langle\eta_{\nu}^{2}\right\rangle / 2}\right. \\
& \left.-\sin [\Omega(\nu+1) \tau] \mathrm{e}^{-\left\langle\eta_{v+1}^{2}\right\rangle / 2}\right\} .
\end{aligned}
$$

Here we have used the fact that the phase difference $\eta_{v} \equiv$ $\Psi(t-v \tau)-\Psi(t)$ has Gaussian distribution with zero mean and therefore $\left\langle\cos \eta_{v}\right\rangle=\exp \left[-\left\langle\eta_{v}^{2}\right\rangle / 2\right]$ and $\left\langle\sin \eta_{v}\right\rangle=0$. With $\eta_{\nu}=-\int_{t-\nu \tau}^{t} \dot{\Psi}(s) \mathrm{d} s$ we obtain for the variance of $\eta_{\nu}$

$\left\langle\eta_{\nu}^{2}\right\rangle=2 \int_{0}^{\nu \tau}(\nu \tau-u) C_{\dot{\psi}}(u) \mathrm{d} u \equiv 2 T_{\nu}$,

where $C_{\dot{\Psi}}(u) \equiv\langle\dot{\Psi}(t) \dot{\Psi}(t+u)\rangle$ is the auto-correlation of the fluctuating part of the frequency. In order to obtain equations for $C_{\dot{\psi}}(u)$, we introduce the auto-correlation of the noise $C_{\xi}(u) \equiv\langle\xi(t) \xi(t+u)\rangle$ and the cross-correlation $C_{\xi \dot{\psi}}(u) \equiv$ $\langle\xi(t) \dot{\Psi}(t+u)\rangle$. After multiplying Eq. (3) with $\dot{\Psi}(t+u)$ or $\xi(t+u)$ and averaging, which is described in detail in Appendix A, we obtain

$$
\begin{aligned}
C_{\dot{\Psi}}(u)= & C_{\xi \dot{\Psi}}(u)+\frac{k}{2} \sum_{\nu=0}^{\infty} R^{\nu}\left\{\cos [\Omega v \tau] \mathrm{e}^{-T_{\nu}} \int_{0}^{\nu \tau} C_{\dot{\Psi}}(s+u) \mathrm{d} s\right. \\
& \left.-\cos [\Omega(\nu+1) \tau] \mathrm{e}^{-T_{\nu+1}} \int_{0}^{(\nu+1) \tau} C_{\dot{\Psi}}(s+u) \mathrm{d} s\right\}, \quad(8) \\
C_{\xi \dot{\Psi}}(u)= & C_{\xi}(u)+\frac{k}{2} \sum_{\nu=0}^{\infty} R^{\nu}\left\{\cos [\Omega v \tau] \mathrm{e}^{-T_{\nu}} \int_{0}^{\nu \tau} C_{\xi \dot{\Psi}}(u-s) \mathrm{d} s\right. \\
& \left.-\cos [\Omega(v+1) \tau] \mathrm{e}^{-T_{\nu+1}} \int_{0}^{(\nu+1) \tau} C_{\xi \dot{\Psi}}(u-s) \mathrm{d} s\right\} .
\end{aligned}
$$

Together with the definition of $T_{v}$ (7), Eqs. (6), (8) and (9) constitute a closed system. To exclude the cross-correlations, we look at the power spectra $S_{\dot{\psi}}(\omega)=\frac{1}{2 \pi} \int_{-\infty}^{\infty} C_{\dot{\psi}}(u) \mathrm{e}^{-\mathrm{i} \omega u} \mathrm{~d} u$ and similar expressions for $S_{\xi}$ and $S_{\xi \dot{\Psi}}$. This leads to

$$
\begin{aligned}
S_{\dot{\Psi}}(\omega)= & S_{\xi \dot{\Psi}}(\omega)+\frac{k}{2} \sum_{\nu=0}^{\infty} R^{\nu}\left\{\cos [\Omega \nu \tau] \mathrm{e}^{-T_{\nu}} S_{\dot{\Psi}}(\omega) \frac{\mathrm{e}^{\mathrm{i} \omega \nu \tau}-1}{\mathrm{i} \omega}\right. \\
& \left.-\cos [\Omega(\nu+1) \tau] \mathrm{e}^{-T_{\nu+1}} S_{\dot{\Psi}}(\omega) \frac{\mathrm{e}^{\mathrm{i} \omega(\nu+1) \tau}-1}{\mathrm{i} \omega}\right\}, \\
S_{\xi \dot{\Psi}}(\omega)= & S_{\xi}(\omega)+\frac{k}{2} \sum_{\nu=0}^{\infty} R^{\nu}\left\{\cos [\Omega \nu \tau] \mathrm{e}^{-T_{v}} S_{\xi \dot{\Psi}}(\omega) \frac{1-\mathrm{e}^{-\mathrm{i} \omega \nu \tau}}{\mathrm{i} \omega}\right. \\
& \left.-\cos [\Omega(\nu+1) \tau] \mathrm{e}^{-T_{\nu+1}} S_{\xi \dot{\Psi}}(\omega) \frac{1-\mathrm{e}^{-\mathrm{i} \omega(\nu+1) \tau}}{\mathrm{i} \omega}\right\} .
\end{aligned}
$$

This system of equations can be solved for $S_{\dot{\psi}}$ :

$$
\begin{aligned}
S_{\dot{\Psi}}(\omega)= & S_{\xi}(\omega)\left[1-\frac{k}{2 \mathrm{i} \omega} \sum_{\nu=0}^{\infty} R^{v} S_{v}\right]^{-1} \\
& \times\left[1+\frac{k}{2 \mathrm{i} \omega} \sum_{\nu=0}^{\infty} R^{v} S_{v}^{*}\right]^{-1} .
\end{aligned}
$$

Here, the star denotes complex conjugation and

$$
\begin{aligned}
S_{\nu} \equiv & \cos [\Omega \nu \tau] \mathrm{e}^{-T_{\nu}}\left(1-\mathrm{e}^{-\mathrm{i} \omega \nu \tau}\right) \\
& \quad-\cos [\Omega(\nu+1) \tau] \mathrm{e}^{-T_{\nu+1}}\left(1-\mathrm{e}^{-\mathrm{i} \omega(\nu+1) \tau}\right) .
\end{aligned}
$$

The spectral form of Eq. (7) reads

$T_{\nu}=\int_{-\infty}^{\infty} S_{\dot{\Psi}}(\omega) \frac{1-\cos [\omega \nu \tau]}{\omega^{2}} \mathrm{~d} \omega$.

Now we make one further approximation: we assume that the spectrum of fluctuations of frequency $\dot{\Psi}$ is very broad, almost white, and replace in (11) the spectrum $S_{\dot{\psi}}(\omega)$ by its value at zero frequency:

$T_{\nu} \approx \int_{-\infty}^{\infty} S_{\dot{\psi}}(0) \frac{1-\cos [\omega \nu \tau]}{\omega^{2}} \mathrm{~d} \omega=\frac{\nu \tau D}{2}$.

In writing the last term we use the Green-Kubo formula $D=$ $2 \pi S_{\dot{\psi}}(0)$, which relates the diffusion constant $D$ of the phase with the power spectrum of the frequency fluctuations at zero frequency. Thus, finally from Eq. (10) we obtain the equation for the diffusion constant

$D=D_{0} \frac{4\left(1+R^{2} E^{2}-2 R E \cos [\Omega \tau]\right)^{4}}{A^{2}(\Omega, \tau, k, R, E)}$.

Here $D_{0}=2 \pi S_{\xi}(0)$ and $E \equiv \mathrm{e}^{-\tau D / 2}$, and

$$
\begin{aligned}
A(\Omega, \tau, k, R, E) & \\
\equiv & E\left(1+R^{2} E^{2}\right)(8 R+k(R-1) \tau) \cos [\Omega \tau] \\
& -2\left(1+4 R^{2} E^{2}+R^{4} E^{4}+k(R-1) R E^{2} \tau\right. \\
& \left.+2 R^{2} E^{2} \cos [2 \Omega \tau]\right) .
\end{aligned}
$$

We also substitute (12) in Eq. (6) which after summation yields

$\Omega=\omega_{0}+\frac{k}{2} \frac{E \sin \Omega \tau(R-1)}{1-2 R E \cos \Omega \tau+R^{2} E^{2}}$.

Eqs. (13) and (14) constitute a closed system for the simultaneous determination of the mean frequency and diffusion, and are the main result of our analysis. In the next section we compare numerical solutions of these implicit equations with direct numerical simulations.

\section{Numerical simulation}

The validity of the analysis above, including that of Gaussian approximation, is now checked by direct simulations of the phase equation (2) assuming the noise $\xi(t)$ to be Gaussian. Clearly, one has to approximate the infinite sum of delay terms. Instead of just truncating this sum at some fixed $v<\infty$, we rather mimic the behavior of real physical systems, starting 

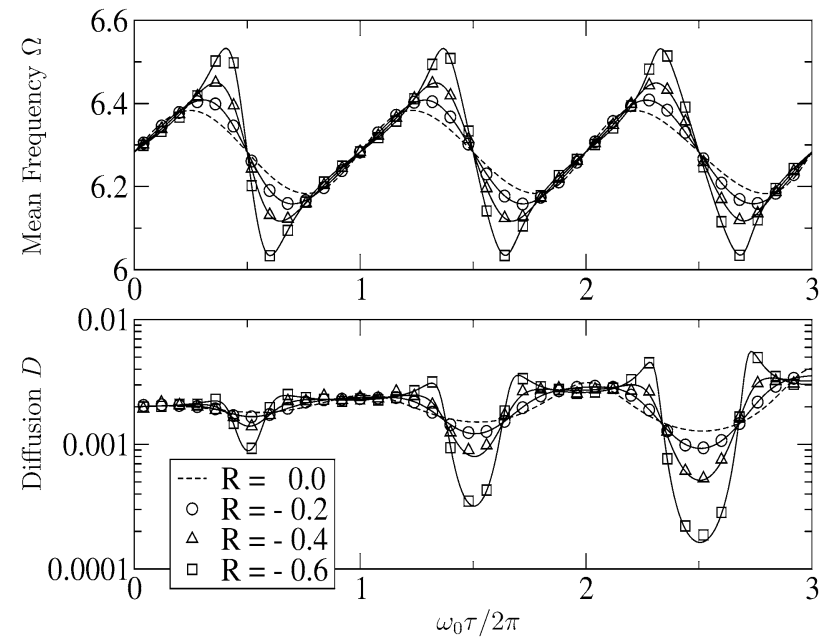

Fig. 2. Diffusion constant $D$ and mean frequency $\Omega$ for small noise $d^{2}=2 \times 10^{-3}$ and $\omega_{0}=2 \pi$ and $k=-0.2$.

at $t=t_{0}$ with given initial condition $\Phi(t) \equiv \Phi_{0}(t)$ for $t \in$ $\left[t_{0}-\tau, t_{0}\right)$ by observing the equality

$\sum_{\nu=0}^{\infty} R^{\nu}\left\{\sin \left[\Phi_{(\nu+1) \tau}-\Phi\right]-\sin \left[\Phi_{\nu \tau}-\Phi\right]\right\}$

$=(1-R)(X \cos \Phi-Y \sin \Phi)$,

where

$X(t) \equiv \sum_{\nu=1}^{\infty} R^{\nu-1} \sin \Phi(t-\nu \tau)=\sin \Phi(t-\tau)+R X(t-\tau)$,

$Y(t) \equiv \sum_{\nu=1}^{\infty} R^{\nu-1} \cos \Phi(t-\nu \tau)=\cos \Phi(t-\tau)+R Y(t-\tau)$.

Note that in order to compute the infinite sum in Eq. (2) now it is only necessary to store the phase and related quantities $X, Y$ over a duration of just one delay period. This successive construction of the infinite sum is completely analogous to the behavior of a signal in a Fabry-Perot interferometer.

In Figs. 2 and 3 we compare the results of the numerical simulations with the analytical predictions according to Eqs. (13) and (14). The correspondence between the numerics and analytical results is very good in the case of small noise (Fig. 2), but less good for stronger noise and high values of $|R|$ and $\tau$ (Fig. 3). Clearly, in comparison to the single delayed feedback scheme of Ref. [5], the introduction of the multiple delayed feedback parameter $R$ can lead to a larger variety of phase dynamics and hence of the system behavior. E.g., for the same value of the feedback strength $k$, with increasing $R$ the suppression of diffusion is stronger.

\section{Conclusion}

In this Letter we have demonstrated that a multiple delay feedback is an effective tool to control coherence of autonomous oscillators. First, such a control scheme enlarges the domain of control parameters where the oscillation frequency remains stable. Analytical calculations of the phase diffusion

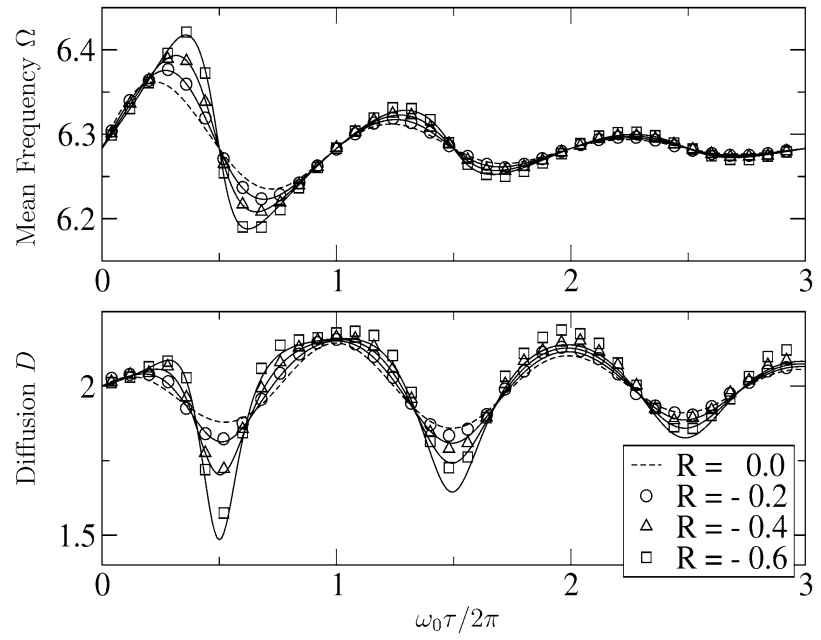

Fig. 3. Diffusion constant $D$ and mean frequency $\Omega$ for large noise $d^{2}=2.0$ and $\omega_{0}=2 \pi$ and $k=-0.2$.

constant have furthermore shown that the range of possible diffusion constant variations is larger for multiple delay scheme compared to the single delay.

The main unresolved question that remains is - what are the properties of the phase dynamics for strong feedback, where several stable oscillation frequencies are possible. Our preliminary numerical calculations show that a transition to multistability is marked by a strong increase of diffusion. A theoretical explanation of this is a subject of future investigations.

\section{Appendix A}

To obtain an equation for $C_{\dot{\Psi}}$, we multiply Eq. (3) by $\dot{\Psi}(t+u)$ and average:

$$
\begin{aligned}
C_{\dot{\Psi}}= & C_{\xi \dot{\Psi}}+\frac{k}{2} \sum_{\nu=0}^{\infty} R^{v}\{\cos [\Omega(v+1) \tau] \\
& \times\left\langle\dot{\Psi}(t+u) \sin \left(\int_{t}^{t-(v+1) \tau} \dot{\Psi}(s) \mathrm{d} s\right)\right\rangle \\
& -\sin [\Omega(v+1) \tau]\left\langle\dot{\Psi}(t+u) \cos \left(\int_{t}^{t-(v+1) \tau} \dot{\Psi}(s) \mathrm{d} s\right)\right\rangle \\
& -\cos [\Omega v \tau]\left\langle\dot{\Psi}(t+u) \sin \left(\int_{t}^{t-v \tau} \dot{\Psi}(s) \mathrm{d} s\right)\right\rangle \\
& \left.+\sin [\Omega v \tau]\left\langle\dot{\Psi}(t+u) \cos \left(\int_{t}^{t-\nu \tau} \dot{\Psi}(s) \mathrm{d} s\right)\right\rangle\right\} .
\end{aligned}
$$

The averages can be calculated using the Furutsu-Novikov formula $[11,12]\langle x F(y)\rangle=\left\langle\frac{\partial F}{\partial y}\right\rangle\langle x y\rangle$, which is valid for Gaussian random variables $x, y$ with zero mean. Then only terms of the type $\langle x \sin y\rangle$ do not vanish:

$$
\left\langle\dot{\Psi}(t+u) \sin \left(\int_{t}^{t-v \tau} \dot{\Psi}(s) \mathrm{d} s\right)\right\rangle=\mathrm{e}^{-T_{v}} \int_{0}^{-v \tau} C_{\dot{\Psi}}(s-u) \mathrm{d} s,
$$




$$
\begin{gathered}
\left\langle\dot{\Psi}(t+u) \sin \left(\int_{t}^{t-(v+1) \tau} \dot{\Psi}(s) \mathrm{d} s\right)\right\rangle \\
=\mathrm{e}^{-T_{v+1}} \int_{0}^{-(v+1) \tau} C_{\dot{\Psi}}(s-u) \mathrm{d} s
\end{gathered}
$$

With these expressions one immediately arrives at Eq. (8). The equation for $C_{\xi \dot{\Psi}}$ can be obtained in a very similar way by multiplying Eq. (3) by $\xi(t+u)$.

\section{References}

[1] K. Pyragas, Phys. Lett. A 170 (1992) 421.

[2] W. Just, H. Benner, E. Schöll, Control of chaos by time-delayed feedback: A survey of theoretical and experimental aspects, in: B. Kramer (Ed.),
Advances in Solid State Physics, vol. 43, Springer, Berlin, 2003, pp. 589603.

[3] M. Rosenblum, A. Pikovsky, Phys. Rev. Lett. 92 (2004) 114102.

[4] M. Rosenblum, A. Pikovsky, Phys. Rev. E 70 (2004) 041904.

[5] D. Goldobin, M. Rosenblum, A. Pikovsky, Phys. Rev. E 67 (2003) 061119.

[6] S. Boccaletti, E. Allaria, R. Meucci, Phys. Rev. E 69 (2004) 066211.

[7] J.E.S. Socolar, D.W. Sukow, D.J. Gauthier, Phys. Rev. E 50 (1994) 3245.

[8] D.W. Sukow, M.E. Bleich, D.J. Gauthier, J.E.S. Socolar, Chaos 7 (1997) 560.

[9] Y. Kuramoto, Chemical Oscillations, Waves and Turbulence, Springer, Berlin, 1984.

[10] A. Pikovsky, M. Rosenblum, J. Kurths, Synchronization. A Universal Concept in Nonlinear Sciences, Cambridge Univ. Press, Cambridge, 2001.

[11] K. Furutsu, J. Res. Natl. Bur. Stand. D 667 (1963) 303.

[12] Y. Novikov, Sov. Phys. JETP 20 (1964) 1290, Zh. Eksp. Teor. Fiz. 47 (1964) 1919. 